\title{
Beobachtungen über das Vorkommen und Laichen des Blauen Wittlings (Micromesistius poutassou Risso) in der Deutschen Bucht
}

\author{
Jürgen Flüchter und Harald Rosenthal \\ Biologische Anstalt Helgoland, Meeresstation, Helgoland \\ Institut für Hydrobiologie und Fischereiwissenschaf, Hamburg
}

\begin{abstract}
Observations on the occurrence and spawning of Couch's Whiting (Micromesistius poutassou Risso) in the German Bight. In 1964 and 1965 a research vessel using a special "shrimp trawl" with various mesh sizes caught several $M$. Poutasson, a fish previously known to occur only in the Atlantic and in the deeper waters of the northern North Sea. Presumably it had not yet been reported in the southern North Sea for two reasons: (a) the large mesh size of the fishing nets used, which may have allowed the fish to slip through, and (b) the failure of fishermen to recognize this rare fish. Two individuals among the 1965 catch were ripe females; one was a ripe male. Successful artificial spawning of these fishes provided an opportunity to study the egg and larval stages of $M$. poutassou. Diameters of eggs ranged from 1.12 to $1.25 \mathrm{~mm}$. Total length of freshly hatched larvae was about $2.2 \mathrm{~mm}$. Incubation time was 11 to 12 days at $8^{\circ} \mathrm{C}$. Measurements of frequency of heartbeat and locomotory activity of embryos at various temperatures were recorded.
\end{abstract}

\section{EINLEITUNG}

Micromesistius poutassou (Syn.: Gadus poutassou) gehört zu den kleineren Gadidenarten. Das eigentliche Verbreitungsgebiet liegt im tiefen atlantischen Wasser (HENDERSON 1957). Die Verbreitungsgrenze scheint sich im Norden im wesentlichen mit der Golfstromdrift zu decken. In der Nordsee ist der Blaue Wittling nur aus den tiefsten Stellen der norwegischen Rinne sowie aus dem äußersten Nordwesten bekannt, soweit diese Gebiete unter $100 \mathrm{~m}$ Tiefe (Sahrhage 1964) und nördlich $57^{\circ} \mathrm{N}$ liegen (SснміDт 1909). In den Jahren 1935 bis 1938 ist dieser Gadide am Nordrande der Nordsee im Beifang der auf Köhler und Makrele ausgerichteten Fischerei sehr zahlreich aufgetreten (LuNDBECK 1942).

Die bis heute unbekannten Eier des Blauen Wittlings, die bisherigen Unsicherheiten über das Alter der Larven, die von den beschriebenen älteren Larvenstadien abweichende Pigmentierung sowie die Vermutung, daß das Auftreten von Micromesistius poutassou in der Deutschen Bucht nicht einmalig ist, waren Veranlassung zur vorliegenden Veröffentlichung. Die Ursachen für das plötzliche Auftreten sind rätsel- 
hafter als beispielsweise das seit dem vorigen Jahr beobachtete Wiedererscheinen des Schellfisches vor Helgoland.

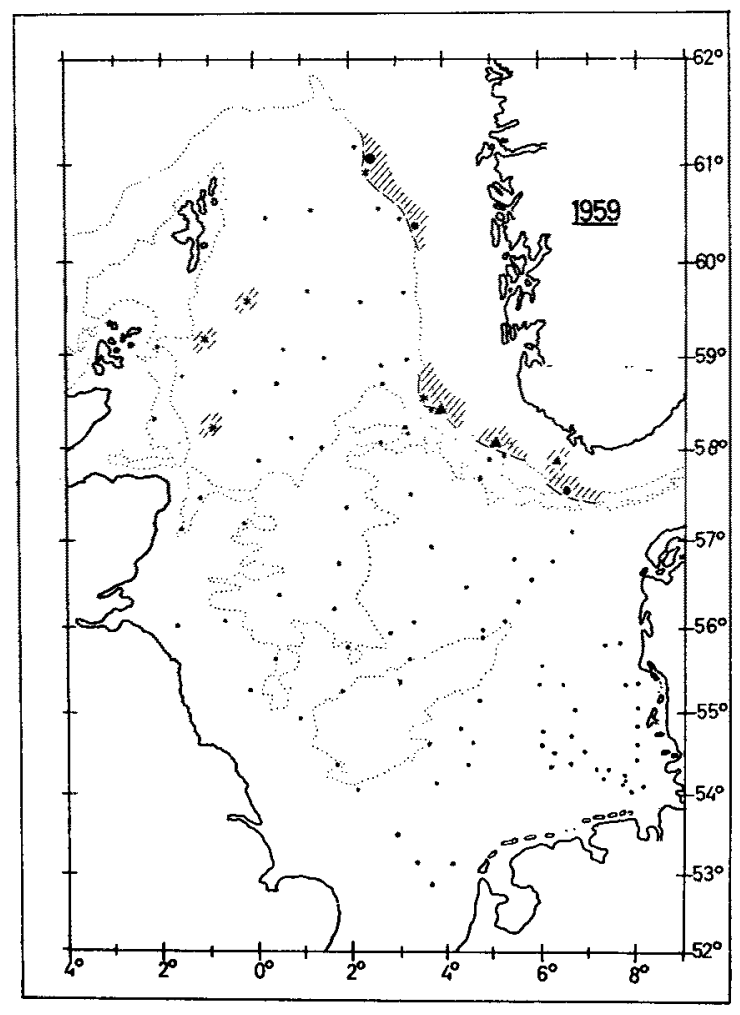

Abb. 1

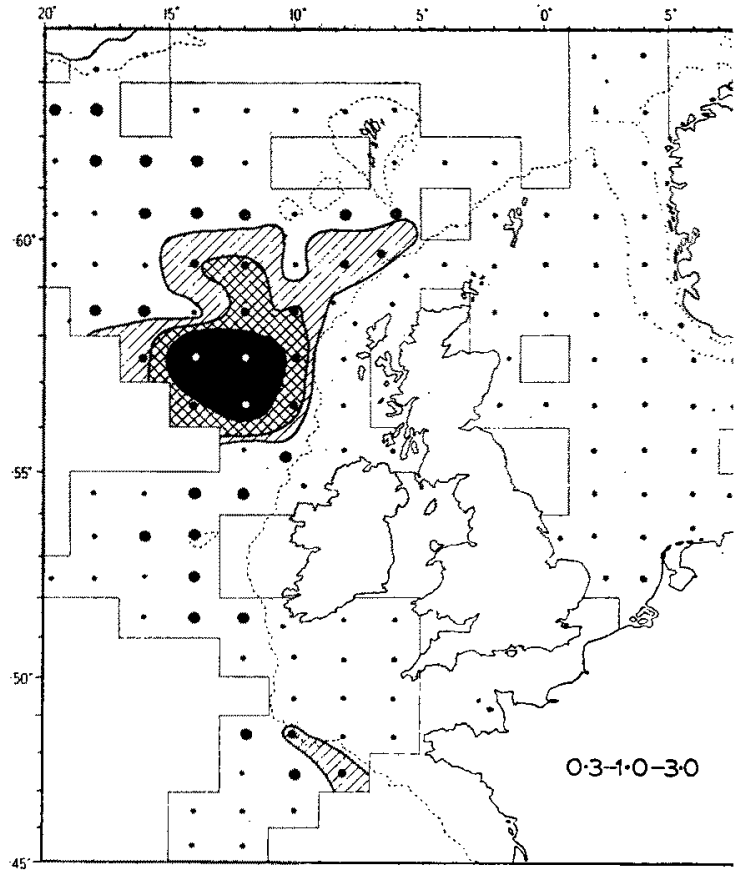

Abb. 2

Abb. 1: Verbreitung des Blauen Wittlings in der Nordsee im Jahre 1959 (nach SAHRHAGE 1964). Die schwarzen Punkte kennzeichnen die Lage der Fischereistationen während der 37. Reise des F.F.S. „Anton Dohrn“. - kein Fang; " 1-9 Fische; 10-49 Fische, $150-99$ Fische Abb. 2: Verbreitung der 0,3 bis $3,0 \mathrm{~cm}$ großen Larven des Blauen Wittlings im NE-Atlantik in den Jahren 1948 bis 1956 (nach Henoerson 1961)

\section{FANGDATEN}

Anfang März 1964 wurden in der Deutschen Bucht (südöstlicher Teil der Helgoländer Rinne, in 50-55 m Tiefe) erstmalig einige Exemplare recht einheitlicher Größe (etwa $16 \mathrm{~cm}$ Totallänge) gefangen. Am 11. März 1965 wurden an der gleichen Stelle drei fast laichreife Tiere (Reifestadium V-VI) und am 29. März drei weitere Exemplare (zwei bereits ausgelaichte $q Q$ und ein noch fließend reifes $\delta$ ) erbeutet. Daraus läßt sich mit Sicherheit folgern, daß Micromesistius poutassou in der Deutschen Bucht nicht nur gelegentlich vorkommt, sondern in diesem Jahr auch abgelaicht hat. Dieses Laichen müßte demnach etwa in der dritten Märzdekade bei Wassertemperaturen zwi- 
schen $3,1^{\circ}$ und $3,8^{\circ} \mathrm{C}$ stattgefunden haben. Am 2. Juni wurden wiederum drei Tiere gefangen (Totallängen: $21 ; 22 ; 22,5 \mathrm{~cm}$ ), die mäßig bis gut gefressen hatten. Im Magen fanden wir neben einigen Crangon allmanni zahlreiche Gammariden, Bodenformen also, die für einen Planktonfresser recht ungewöhnlich sind.

\section{EIER UND LARVEN}

Von den am 11. März gefangenen Tieren wurden die Gonaden herauspräpariert, und es gelang, nach dem Verrïhren der Geschlechtsprodukte etwa 20 befruchtete Eier zu isolieren. Die Eigrößen lagen zwischen 1,12 und 1,25 $\mathrm{mm}^{1}$. Durch die methodisch bedingte starke bakterielle Kontamination war die Eisterblichkeit schon in den ersten Tagen recht hoch. Trotz der geringen Eizahl hielten wir es jedoch für wichtig, die Beobachtungen fortzusetzen, da über die Fortpflanzungsbiologie dieser Art nichts bekannt ist.

Die Eier wurden bei konstant $8^{\circ} \mathrm{C}$ und $35 \% \mathrm{~S}$ gehalten. Vier Tage nach der Befruchtung wurde der Keimstreifen sichtbar, am sechsten Tag war er deutlich ausgeprägt und umspannte den Dotter unter einem Winkel von 110 bis $120^{\circ}$. Die Inkubationszeit betrug etwa 11 bis 12 Tage. Das Schlüpfen erstreckte sich über 2 Tage. Auffallend war, daß die zunächst aufschwimmenden befruchteten Eier etwa einen Tag vor

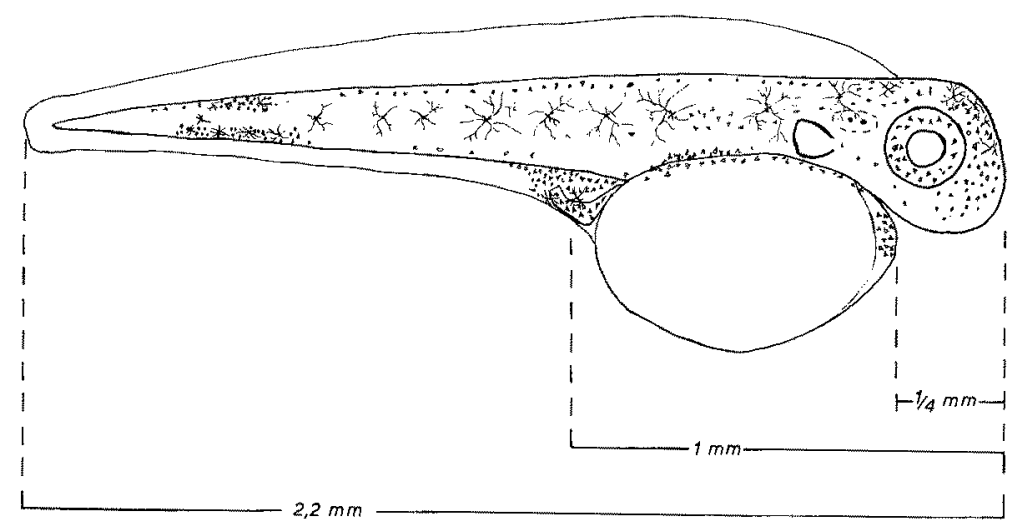

Abb. 3: Larve von Micromesistius pontasson Risso (5 Tage alt; $2,2 \mathrm{~mm}$ lang). Die kleinkörnigen Pigmente sind gelb bis orangefarben, die dendritenförmigen schwarz

dem Schlüpfen zum Boden absanken. Zwei bis drei Tage vorher waren die ersten schwarzen Chromatophoren zu erkennen. Die Pigmentierung der beim Schlüpfen ungefähr 2,2 mm langen Larven weicht von den bisherigen Angaben verschiedener Autoren ab. Bereits einen Tag vor dem Schlüpfen besitzt die Larve, lateral in gleichen $\mathrm{Ab}$ ständen angeordnet, etwa neun sehr stark verzweigte, dendritenförmige Chromatophoren. Etwa zwei Tage nach dem Schlüpfen treten kleinkörnige, gelb bis orangefar-

${ }^{1}$ Die in Bourn fixierten Eier messen nach 6 Wochen 0,92 bis $1,00 \mathrm{~mm}$ im Durchmesser. 
bene Pigmente gleichmäßig über den Körper verteilt auf; lediglich am hinteren Ende des Dottersacks und kurz vor dem Rumpfende sind Anhäufungen festzustellen. Der Dottersack bleibt unpigmentiert und ist noch sehr groß. Die Mundöffnung wird selbst am füntten 'Tag nach dem Schlüpfen noch nicht sichtbar. Die Augen waren unpigmentiert.

Tabelle 1

Zeitlicher Ablauf der Embryonalentwidklung bei $8^{\circ} \mathrm{C}$. Beobachtungen an 5 Larven; 2 davon starben kurz nach dem Schlüpfen

\begin{tabular}{|c|c|c|}
\hline $\begin{array}{l}\text { Datum } \\
(1965)\end{array}$ & $\begin{array}{c}\text { Tage nach der } \\
\text { Befruchtung }\end{array}$ & Entwicklungszustand \\
\hline 11. März & 0 & Befruchtung \\
\hline 15. & 4 & Keimstreifen erkennbar \\
\hline 17. & 6 & $\begin{array}{l}\text { Keimstreifen mit deutlich differenzierter } \\
\text { Kopfpartie }\end{array}$ \\
\hline 20. & 9 & Schwarze Chromatophoren \\
\hline $22 . / 23$ & $11 / 12$ & Larven schlïpfen \\
\hline 26. & 14 & Auftreten gelben Pigments \\
\hline 29. & 17 & letzte Larve + \\
\hline
\end{tabular}

Der günstige Zufall, die Embryonen einer hochatlantischen Form im Zusammenhang mit anderen noch laufenden Untersuchungen an Fischbrut zu beobachten, erbrachte die Möglichkeit, Anhaltspunkte dafür zu sammeln, ob der in der Deutschen Bucht abgelegte Laich unter den hier gegebenen Umweltbedingungen - insbesondere den in dieser Jahreszeit niederen Temperaturen - Aufwuchschancen hat. Einige Eier wurden zu diesem $Z$ weck etwa drei Tage vor dem Schlüpfen von den übrigen getrennt gehältert und die Wassertemperatur zunächst langsam auf $3^{\circ} \mathrm{C}$ abgekühlt. Nach einer dreistündigen Anpassungszeit wurde dann im Verlauf von 12 Stunden auf $10^{\circ} \mathrm{C}$ stufenweise aufgeheizt. Dabei wurden Beobachtungen über Herzschlagfrequenz und Embryoaktivität protokolliert.

Als Maßzahl für den Herzschlag dient die Anzahl der Sekunden, innerhalb derer das Herz $50 \mathrm{mal}$ schlägt $\left(\mathrm{n}=\mathrm{T}_{50}\right)$. Wie auch von anderen Fischarten bekannt (vgl. WICKLER 1957) hängt der Herzschlag der Embryonen gegen Ende der Inkubationszeit

Tabelle 2

Herzschlagfrequenzen eines Embryos von Micromesistius poutassou bei verschiedenen Temperaturen (2-3 Tage vor dem Schlüpfen). $T_{50}=$ Zeit pro 50 Schläge; $\mathrm{T}_{50}=$ Mittelwert aus drei Messungen; $\triangle \mathrm{T}_{50}=$ Frequenzdifferenz

\begin{tabular}{|ccccccc|}
\hline $\begin{array}{c}\text { Wasser- } \\
\text { temperatur }\end{array}$ & $3,5^{\circ} \mathrm{C}$ & $4,5^{\circ} \mathrm{C}$ & $7,0^{\circ} \mathrm{C}$ & $8,0^{\circ} \mathrm{C}$ & $8,5^{\circ} \mathrm{C}$ & $9,0^{\circ} \mathrm{C}$ \\
\hline $\mathrm{T}_{50}$ & $2^{\prime} 13^{\prime \prime}$ & $2^{\prime} 04^{\prime \prime}$ & $1^{\prime} 55^{\prime \prime}$ & $1^{\prime} 36^{\prime \prime}$ & $1^{\prime} 18^{\prime \prime}$ & $1^{\prime} 05^{\prime \prime}$ \\
& $2^{\prime} 06^{\prime \prime}$ & $2^{\prime} 07^{\prime \prime}$ & $1^{\prime} 53^{\prime \prime}$ & $1^{\prime \prime} 35^{\prime \prime}$ & $1^{\prime \prime} 19^{\prime \prime}$ & $1^{\prime \prime} 06^{\prime \prime}$ \\
& $2^{\prime} 09^{\prime \prime}$ & $2^{\prime} 02^{\prime \prime}$ & $1^{\prime} 59^{\prime \prime}$ & $1^{\prime} 39^{\prime \prime}$ & $1^{\prime} 16,5^{\prime \prime}$ & $1^{\prime} 06,5^{\prime \prime}$ \\
\hline $\mathrm{T}_{50}$ & $2^{\prime} 09,3^{\prime \prime}$ & $2^{\prime} 04,3^{\prime \prime}$ & $1^{\prime} 55,6^{\prime \prime}$ & $1^{\prime} 36,6^{\prime \prime}$ & $1^{\prime} 17,8^{\prime \prime}$ & $1^{\prime} 05,7^{\prime \prime}$ \\
\hline$\triangle \mathrm{T}_{50}$ & $7,0^{\prime \prime}$ & $5,0^{\prime \prime}$ & $6,0^{\prime \prime}$ & $4,0^{\prime \prime}$ & $2,5^{\prime \prime}$ & $1,5^{\prime \prime}$ \\
\hline
\end{tabular}


nur von der Wassertemperatur und kaum von der Häufigkeit der Bewegungen des Embryos ab. Die Herzschlagfreqenzen können jedoch individuell schwanken. Um vergleichbare Werte zu erzielen, wurden die Messungen bei allen Temperaturen stets an dem gleichen Individuum vorgenommen.

In Tabelle 2 sind die ermittelten Werte zusammengestellt. Auffallend ist, daß die individuellen Frequenzdifferenzen unter Berücksichtigung der Beobachtungsintervalle bei zunehmender Temperatur stetig abnehmen (siehe Tab. 2 und Abb. 4). Allerdings

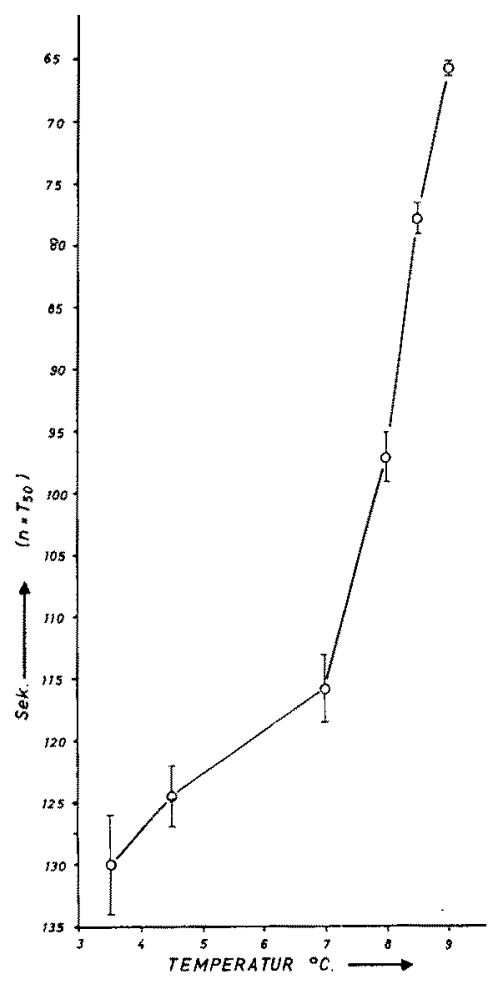

Abb. 4

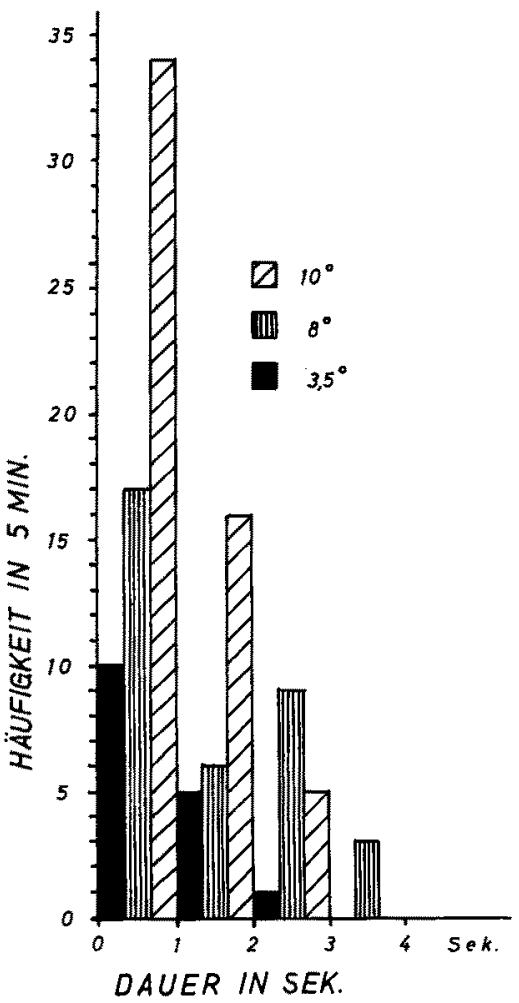

Abb. 5

Abb. 4: Herzschlagfrequenzen bei verschiedenen Temperaturen (nach den Daten der Tab. 2) Abb. 5: Häufigkeitsverteilung und Dauer der Embryobewegungen pro 5 Minuten bei $3,5^{\circ}, 8^{\circ}$ beziehungsw eise $10^{\circ} \mathrm{C}$

kann aus den Daten nicht ohne weiteres abgelesen werden, ob die auftretenden geringeren Differenzen auf die erhöhte Schlagzahl oder auf eine regelmäßigere Herztätigkeit zurückzuführen sind. Der Wert $\bar{T}_{50}$ beträgt bei $9^{\circ} \mathrm{C}$ gegenüber dem Ausgangswert bei 3,50 $\mathrm{C}$ nur noch $50 \%$. Entsprechend dürfte $\triangle T_{\tilde{5} 0}^{*}$ um $50 \%$ abnehmen, ohne daß sich damit die individuelle Frequenzdifferenz geändert hat.

Die Häufigkeit der Embryobewegungen ist ebenfalls temperaturabhängig. Es handelt sich dabei um sehr schnelle, von vorn nach hinten über die Körperachse verlaufende 
sinusförmige Wellenbewegungen, die sehr an echte Schwimmbewegungen erinnern. Die Dauer der einzelnen Bewegungsphasen erreicht maximal 4 Sekunden, am häufigsten sind Bewegungen unter 1 Sekunde (meist sogar unter 0,5 Sek.). Die Aktivitätszunahme ist bei Temperaturerhöhung am deutlichsten bei den kurzen Bewegungsphasen zu beobachten. Mit steigender Temperatur nahm die Länge der Ruhepausen zwischen den einzelnen Bewegungsphasen zugunsten der kurzen Aktivitätsphasen ab.

Die vorhandenen Werte wurden an geringem Material gewonnen und sind möglicherweise nicht reproduzierbar. Sie unterstützen jedoch die Vermutung, daß ein Aufkommen der Brut unter den Bedingungen im Seegebiet um Helgoland im Frühjahr dieses Jahres unwahrscheinlich ist. Die Wassertemperaturen lagen während der Laichzeit zwischen $3,1^{\circ}$ und 3,8 $\mathrm{C}$ in Bodennähe und 1,10 (5. März 1965) bis 4,80 (8. April $1965)$ an der Oberfläche. Die Embryoaktivität ist bei diesen Temperaturen sicher als abnorm gering zu bezeichnen.

\section{DISKUSSION}

Die Beobachtungen über den Zeitpunkt des Laichens und das Aussehen der Larven lassen den Verdacht aufkommen, daß die bisher beschriebenen und bekannten Larven von M. poutassou nicht die jüngsten Stadien sind. Ehrenbaum (1909) beschreibt Larven mit vollpigmentierten Augen, ohne Dottersack und mit gut ausgebildetem Maul; ebenso Henderson (1961), der die entsprechenden Längen mit 2,7 bis 3,0 mm angibt.

Unsere Kenntnisse über diese Art sind noch sehr gering, und die Aussagen über Eier, Larven und Jungfische basieren auf sehr lückenhaftem Material. Die Eier waren bis heute unbekannt. HENDERSON (1957) glaubt lediglich eine leere Eihülle gefunden zu haben. Von den einzelnen Autoren sind wiederum nur Larven mit Längen zwischen $3 \mathrm{~mm}$ und $30 \mathrm{~mm}$ beschrieben worden. Die kleinsten bekannten Jungfische sind jedoch $8 \mathrm{~cm}$ und größer ${ }^{2}$. Zwei deutliche Lücken wurden also durch Interpolation überbrückt. Der sichere Weg vom adulten laichreifen Tier über das Ei zur Larve konnte bisher nicht beschritten werden. Dies lag daran, daß man bis heute keinen Laichschwärmen begegnet ist. Es ist nichts Direktes über Laichzeit und Lage der Laichplätze bekannt. Bislang fehlen Beobachtungen, da die kommerzielle Fischerei auf andere Fischarten ausgerichtet ist und das Hauptvorkommen nicht direkt auf den Fanggrinden liegt.

Obwohl das Gebiet um Helgoland sehr intensiv befischt wird, sind Fangmeldungen bisher nie bekannt geworden. Das Vorkommen des Blauen Wittlings in diesem Raum ist in den letzten beiden Jahren jedoch sicher häufiger, als die spärlichen Fangdaten belegen. Der geringe Körperquerschnitt dieses Fisches ermöglicht ihm ein leichtes Entkommen aus dem hier gebräuchlichen, vornehmlich auf Plattfisch und Kabeljau ausgelegten Kuttertrawl. Darüber hinaus ist der Blaue Wittling den meisten Fischern unbekannt und dürfte - selbst wenn er im Beifang vorhanden ist - somit unerkannt bleiben.

Unsere Fänge wurden vom Forschungskutter „Uthörn“ mit dem engmaschigen

2 BoLDowskr (1939) hat allerdings einige Exemplare zwischen 5 und $15 \mathrm{~cm}$ gefangen (zitiert nach HENDERSON 1957). 
"Helgoländer Trawl" (einer modifizierten Garnelenkurre) erzielt". Die Schleppzeit setrug wegen des rauhen Untergrundes an dieser Stelle jeweils nur 6 bis 8 Minuten.

\section{ZUSAMMENFASSUNG}

1. Micromesistius poutassou Risso wurde erstmals im März 1964 und mehrmals im Frühjahr 1965 bei Helgoland gefangen.

2. Der Fang fast laichreifer Tiere ermöglichte die Erbrütung der bislang unbekannten Eier.

3. Es werden Angaben über Entwicklungsdauer der Eier und Pigmentierung der Larven gemacht: Die Eidurchmesser liegen bei 1,12 bis $1,25 \mathrm{~mm}$; die frischgeschlüpften Larven sind etwa 2,2 mm lang; die Inkubationszeit beträgt bei $8^{\circ} \mathrm{C} 11$ bis 12 Tage.

4. Messungen über Herzschlagfrequenzen und Aktivität der Embryonen wurden bei verschiedenen Temperaturen durchgeführt und diskutiert.

\section{ZITIERTE LITERATUR}

Ehrenbaum, E., 1909. Eier und Larven von Fischen. In: Nordisches Plankton. Zoologischer Teil. Lipsius \& Tischer, Kiel, Bd 1, 413 pp.

Henderson, G. T. D., 1957. The distribution of young Gadus pontasson (Risso). Bull. mar. Ecol. 4 (35), 179-202.

- 1961. Contributions towards a plankton atlas of the north-eastern Atlantic and the North Sea. Bull. mar. Ecol. 5 (42), 105-111.

Lundieck, J., 1942. Die Ertragsstatistik der deutschen Dampfhochseefischerei für die Jahre 1935-1938. Ber, dt. wiss, Komm. Meeresforsch. NF 11 (1), 1-29.

Sarrhage, D., 1964. Uber die Verbreitung der Fischarten in der Nordsee. Q. Juni-Juli 1959 und Juli 1960. Ber. dt. weiss. Komm. Meeresforsch. NF 17 (3), 190-246.

ScHmidt, J., 1909. The distribution of the pelagic fry and the spawning regions of the gadoids in the North Atlantic from Iceland to Spain. Rapp. P.,v. Rétn. Cons. perm. int. Explor. Mer 10 (4), 1-229.

WI ICKLER, W., 1957. Die Larve von Blennius fluviatilis Asso 1784. Biol. Zbl. 76, 453-466.

3 Herrn Kapitän Hornsmann danken wir herzlich für seine Aufmerksamkeit bei de Durchsicht der Fänge. 